\title{
Managing Prolonged Pain After Surgery: Examining the Role of Opioids.
}

\author{
Eric S. Schwenk \\ Thomas Jefferson University \\ John-Paul J. Pozek \\ University of Kansas Medical Center \\ Eugene R. Viscusi \\ Thomas Jefferson University
}

Follow this and additional works at: https://jdc.jefferson.edu/anfp

Part of the Anesthesiology Commons

Let us know how access to this document benefits you

\section{Recommended Citation}

Schwenk, Eric S.; Pozek, John-Paul J.; and Viscusi, Eugene R., "Managing Prolonged Pain After Surgery: Examining the Role of Opioids." (2018). Department of Anesthesiology Faculty Papers. Paper 45.

https://jdc.jefferson.edu/anfp/45

This Article is brought to you for free and open access by the Jefferson Digital Commons. The Jefferson Digital Commons is a service of Thomas Jefferson University's Center for Teaching and Learning (CTL). The Commons is a showcase for Jefferson books and journals, peer-reviewed scholarly publications, unique historical collections from the University archives, and teaching tools. The Jefferson Digital Commons allows researchers and interested readers anywhere in the world to learn about and keep up to date with Jefferson scholarship. This article has been accepted for inclusion in Department of Anesthesiology Faculty Papers by an authorized administrator of the Jefferson Digital Commons. For more information, please contact: JeffersonDigitalCommons@jefferson.edu. 


\begin{abstract}
A notable minority of patients experience persistent postsurgical pain and some of these patients consequently have prolonged exposure to opioids. Risk factors for prolonged opioid use after surgery include preoperative opioid use, anxiety, substance abuse, and alcohol abuse. The window to intervene and potentially prevent persistent opioid use after surgery is short and may best be accomplished by both surgeon and anesthesiologist working together. Anesthesiologists in particular are well positioned in the perioperative surgical home model to affect multiple aspects of the perioperative experience, including tailoring intraoperative medications and providing consultation for possible discharge analgesic regimens that can help minimize opioid use. Multimodal analgesia protocols reduce opioid consumption and thereby reduce exposure to opioids and theoretically the risk of persistent use. Regional anesthesia and analgesia techniques also reduce opioid consumption. Although many patients will recover without difficulty, the small minority who do not should receive customized care which may involve multiple office visits or consultation of a pain specialist. Enhanced recovery pathways are useful in optimizing outcomes after surgery.
\end{abstract}

Key Words: Total joint arthroplasty; persistent opioid use after surgery; multimodal analgesia; opioid epidemic; persistent post-surgical pain 


\section{Introduction}

While many patients will follow a normal and expected course of recovery from pain following joint replacement surgery, a portion of patients will present with severe pain of longer duration often leading to prolonged opioid exposure. These patients are at risk of developing long term opioid use and potentially substance use disorder. By specific evaluation prior to surgery and the incorporation of opioid-reducing strategies preoperatively, intraoperatively and postoperatively within the context of enhanced recovery pathways, we may best address the needs of these challenging patients. Orthopaedic surgeons and their colleagues in orthopaedic anesthesia have been early adopters of enhanced recovery pathways and have realized many benefits for their patients. However, these pathways often neglect patients who are outliers and who need customized analgesic approaches. We will present the special considerations for these patients. The best treatment is likely prevention followed by early intervention.

The effect of patient and surgical characteristics on the risk of persistent opioid use after surgery in opioid-nä̈ve patients

Although not every patient taking opioids persistently beyond a month after surgery can be predicted, there are several common characteristics that can be discussed in further detail. Sun et al [1] performed a retrospective database analysis using administrative claims data over a 12year period. A group of opioid-naïve patients who underwent one of 11 different surgery types was compared to a matched cohort of opioid-naïve patients who did not undergo surgery. The incidence of chronic opioid use after total knee arthroplasty (TKA) according to their definition was $1.41 \%$ compared to $0.136 \%$ in their control sample. Among demographic factors they found 
that both male gender and age greater than 50 years were associated with increased odds of taking chronic opioids, defined as having 10 or more opioid prescriptions or taking opioids for more than 120 days (excluding the first 90 postoperative days) during the first year after surgery. The association with male gender may seem somewhat surprising as previous studies have linked the risk of chronic postsurgical pain (CPSP) to younger females [2,3]. However, this is a key distinction, that is, the difference between CPSP and chronic opioid use. Although some patients who develop CPSP ultimately will end up taking chronic opioids, this is not always the case and the patient factors may be different.

Several other patient factors were identified by Sun et al [1], including preoperative drug abuse, alcohol abuse, depression, as well as use of medications including benzodiazepines and antidepressants. Similarly, Brummett et al [4] in another large database study of surgical patients found that preoperative tobacco use, alcohol and substance use disorders, anxiety, and pain disorders were associated with chronic opioid use after surgery. In their study, the incidence of persistent opioid use was more liberally defined as the filling of one opioid prescription between 90 and 180 days. Not surprisingly their reported incidence of persistent opioid use was higher than that of Sun et al (about $6 \%$ for Brummett et al). It should also be noted that Brummett et al did not include any major orthopedic procedures in their cohort.

One might surmise that more painful surgeries would be more likely to result in persistent opioid use. Although Sun et al [1] found this to be the case, Brummett et al [4] found no difference in persistent opioid use between major and minor surgery. The patient risk factors stated above, including anxiety, alcohol and substance use disorder, suggest that persistent surgical pain alone is not the driving force behind abnormally prolonged opioid use. Many of the reasons have yet to be elucidated. The implications of Brummett et al, namely that minor surgery 
resulted in just as high incidence of persistent opioid use as major surgery, are concerning. If that is indeed the case then there is an effect of opioid molecules themselves, at least in susceptible patients, that is powerful enough that even minor surgery can lead to prolonged use.

Patient characteristics associated with persistent opioid use after total knee arthroplasty in opioid-tolerant patients

Patients taking opioids chronically prior to surgery represent a completely different scenario and present a perioperative challenge as they are more likely to consume higher doses of opioids and rate their pain intensity higher in the first $48 \mathrm{~h}$ after TKA than their opioid-naïve counterparts [5]. Namba et al [6] retrospectively studied 23,726 patients who underwent TKA in the Kaiser Permanente system; $60 \%$ of the patients in this cohort were taking opioids at the time of surgery. However, although they did report co-morbidities that included $11.6 \%$ with chronic back pain and $13.6 \%$ with nonspecific chronic pain, the diagnoses for which patients were taking preoperative opioids were not available. At 3 months after surgery $41.2 \%$ of patients were still taking opioids; this dropped to $30 \%$ at $9-12$ months postoperatively. This is more than 5 times the rate of persistent opioid use reported by Brummett et al at the same time postoperatively [4].

Preoperative use of opioids is consistently shown to be linked to persistent opioid use after surgery. A large database study of patients who underwent upper extremity surgery revealed that patients who had received opioids prior to surgery were more likely to fill opioid prescriptions and receive refills after surgery [7]. In a study of a mixed surgical population, preoperative opioid use was once again a risk factor for persistent use [8]. As the preoperative opioid daily dose increases, the risk of persistent use also increases [9]. Possible patient risk factors for persistent opioid use are shown in Table 1. 
Does intraoperative management affect the risk of persistent opioid use after surgery?

Anesthesiologists can and should play a key role in guiding patients through the perioperative experience. The Perioperative Surgical Home (PSH) model of care, which is a "patient-centered and physician-led multidisciplinary and team-based system of coordinated care that guides the patient throughout the entire surgical experience [10]," is one way to empower anesthesiologists in making decisions that positively impact patient care. Anesthesiologists have the training and experience to evaluate patient disease and create a perioperative plan best for each patient. The PSH will be discussed in more detail in the following sections. When it comes to intraoperative management, there is evidence that even brief exposure to high doses of remifentanil, a potent, very short-acting opioid, is associated with chronic pain [11]. Chia et al [12] demonstrated that high-dose intraoperative fentanyl can lead to greater postoperative pain intensity and opioid consumption, including the induction of acute opioid tolerance. Opioidinduced hyperalgesia can develop rapidly, including during the perioperative period when relatively brief periods of opioid exposure occur [13]. The use of regional anesthesia for total joint arthroplasty (TJA) is one way to reduce perioperative opioid use as well as morbidity and should be considered for all patients without contraindications. Patients who undergo TJA with regional anesthesia (neuraxial or peripheral nerve block) have improved postoperative pain and fewer opioid-related adverse effects, presumably from reduced opioid consumption [14]. Regional anesthesia also may reduce the incidence of surgical site infections, pulmonary complications, and length of stay $[15,16]$. Anesthesiologists are in an ideal position to recommend regional anesthesia and help develop clinical protocols that feature it. This has been advocated by some as a component of an effective multimodal analgesic protocol for TKA [17]. 
Role of Enhanced Recovery Pathways in Reducing Opioid Usage

Efforts to improve perioperative care of patients using evidence-based medicine has led to the implementation of protocols that minimize opioid usage. Adopted in the United Kingdom and Europe, enhanced recovery after surgery (ERAS) protocols use multidisciplinary pathways designed to standardize care and apply evidence-based medicine to all phases of the perioperative period [18].

Opioid reduction via use of multimodal analgesics, peripheral nerve blocks, and neuraxial analgesia is often included to help meet recovery and discharge goals. Use of these techniques in ERAS protocols has been associated with early return of bowel function [19], less overall complications, and earlier discharge after colorectal surgery [20]. A study by Scott et al [21] demonstrated that the use of spinal anesthesia in an ERAS protocol for TJA was associated with earlier mobilization and decreased postoperative stay by 1 day.

Implementation of ERAS pathways has met resistance. Protocols are becoming more complex, with a variable amount of supportive evidence for each element [22]. While studies show that strict adherence an ERAS protocol leads to a lower risk of postoperative complications and postoperative symptoms that delay discharge, compliance varies, especially in the postoperative period [23].

In the United States, hospitals and national organizations including the American Society of Anesthesiologists (ASA) have advocated adopting the PSH model. Both ERAS and PSH protocols are designed to decrease variability in perioperative care and can reduce opioid consumption in the perioperative period. One difference is the PSH is more patient-centered, allowing for patient-specific customization in treatment of challenging patient groups [24]. 
Within the PSH framework, custom analgesic plans can be formulated for patients with chronic pain, particularly those who are medically complex or opioid tolerant.

\section{Reducing Opioid Prescriptions}

Careful consideration of an analgesic regimen to treat acute pain is important in the inpatient period and similar principles should be applied upon patient discharge. The Centers for Disease Control and Prevention (CDC) recognize that chronic opioid use often begins with treatment of acute pain [25]. This is reflected in current prescription guidelines stating that "treatment of acute pain with opioids should be for the shortest duration possible" [26]. Ideally, clinicians should prescribe the lowest effective dose of an immediate-release opioid with a duration less than three days and no more than seven days.

Encouraging trends in opioid prescriptions were observed in the past few years [27]. From 2014-16, prescriptions decreased 5\% annually. The number of patients taking opioids per 100 Americans has dropped from 20.7 to 19.1 and the average morphine milligram equivalent has decreased from 59.7 to 47.1. However, the number of patients per 100 Americans receiving chronic pain prescriptions (prescription with an opioid supply $>30$ days) has risen sharply in the past 10 years.

A study from Levy et al [28] evaluating opioid prescriptions by medical specialties from 2007-12 showed that specialties associated with the treatment of painful conditions were more likely to prescribe opioids. While overall prescription rates rose in this time period, they stabilized after 2010. Specialties more likely to manage chronic pain, such as pain management, physical medicine/rehabilitation, internal medicine and family medicine, experienced steady increases in opioid prescriptions (Figure 1). Other specialties associated more with acute pain 
conditions, such as surgeons, emergency medicine physicians, and dentists, decreased prescription rates before and after 2010 .

After the declaration of the opioid crisis as a 'national emergency,' external entities have taken measures to decrease the prescription and consumption of these medications. The Drug Enforcement Administration mandated a 20\% reduction in opioid manufacturing for 2018 [29]. CVS, the largest retail pharmacy company in the U.S., has recently changed its opioid dispensing guidelines [30]. Opioid-naïve patients with acute pain are limited to a 7-day supply of the medication. Daily dosage will be limited based on the strength of the opioid, and they will require use of immediate-release opioids before dispensing the extended-release formulation.

The effect of duration of opioid prescription after surgery and risk of persistent use

The duration of opioid prescription a patient initially receives after surgery has a major impact on the likelihood he or she is still taking opioids months to years later. In its weekly morbidity and mortality report the CDC presented data from 2006-2015 showing that patients who received an initial opioid prescription for 10 days had almost a $20 \%$ probability of still taking opioids 1 year later. That probability increased to about 35\% at 1 year with a 30 -day supply [26]. From that same report, it is clear that the number of prescriptions in the first episode of opioid use increases the probability of taking opioids at both 1 and 3 years later, as does starting a long-acting opioid for the patient as part of the initial prescription. The data included all opioid prescriptions and not just surgical patients, so the population may not represent surgical patients or orthopedic patients. However, the message is clear that patients should be sent home after surgery with the shortest supply of opioids necessary. 


\section{Monitoring Opioid Use After Discharge}

Acute pain can be multifactorial with nociceptive, visceral, neuropathic, inflammatory or muscle spasm components [31]. No one medication can treat all components. Formulation of an optimal analgesic regimen after discharge should cover specific agents to treat these components, the necessity of opioids, and expected duration of opioid usage.

In the United States, postoperative analgesic prescriptions are traditionally written by surgeons. However, providing adequate analgesia in the perioperative period and after discharge is the responsibility of the entire perioperative team. Communication about the optimal analgesic regimen, monitoring the postoperative pain trajectory (Table 2), and post-discharge opioid use should occur between the surgeon and anesthesiologist or acute pain physician.

\section{Conclusion}

Persistent pain following joint replacement surgery will occur in a subset of patients placing them at risk for prolonged opioid use and potential long-term opioid exposure and potential substance use disorder. The exact number following arthroplasty is not known but overall numbers in general surgery suggest $6-10 \%$ will be using opioids at one year following their procedure. The adoption of enhanced recovery pathways minimizing or eliminating opioids should be considered standard practice. Furthermore, by carefully identifying potential outliers for persistent pain and applying customized care, we may further reduce the potential for persistent opioid use. Clearly, the use of opioids as first-line or primary analgesics for perioperative pain must be reconsidered. 
Figure legend

Figure 1. Opioid prescriptions from 2007-2012 by specialty 
Table 1. Risk factors for persistent opioid use after surgery

\begin{tabular}{|c|}
\hline Likely \\
\hline Preoperative opioid use \\
\hline Anxiety \\
\hline Substance abuse \\
\hline Alcohol abuse \\
\hline Possible \\
\hline Tobacco use \\
\hline Antidepressant use \\
\hline
\end{tabular}


Table 2. Recommendations for monitoring the postoperative pain trajectory to ensure minimal opioid usage and proposed interventions in the case of persistent opioid use.

\begin{tabular}{|c|c|c|}
\hline Phase of Care & Monitoring & Intervention \\
\hline Hospital Course & History and physical & $\begin{array}{l}\text { Anesthetic/analgesic plan that } \\
\text { minimizes opioid use }\end{array}$ \\
\hline Time of Discharge & History and physical & $\begin{array}{ll}\text { - } & \text { Multimodal analgesia } \\
\text { - } & \text { Continuous peripheral nerve } \\
\text { blocks (if indicated) } \\
\text { - } \\
\text { Prescription for 5-7 days of } \\
\text { smallest dose of immediate- } \\
\text { release oral opioids }\end{array}$ \\
\hline Post-discharge (5-7 days) & $\begin{array}{c}\text { Office Visit } \\
\text { Telemedicine encounter } \\
\text { Phone call }\end{array}$ & $\begin{array}{l}\text { - If pain controlled, continue } \\
\text { with non-opioid medications } \\
\text { If pain uncontrolled, consider } \\
\text { intervention: adding non- } \\
\text { opioid medications, } \\
\text { mindfulness, meditation, } \\
\text { consultation with expert in } \\
\text { pain management }\end{array}$ \\
\hline Post-discharge (3 weeks) & $\begin{array}{c}\text { Office visit } \\
\text { Telemedicine encounter } \\
\text { Phone call }\end{array}$ & $\begin{array}{l}\text { - Aggressive intervention } \\
\text { - Consultation with expert in } \\
\text { pain management }\end{array}$ \\
\hline
\end{tabular}




\section{References}

1. Sun EC, Darnall BD, Baker LC, Mackey S. Incidence of and risk factors for chronic opioid use among opioid-naive patients in the postoperative period. JAMA Intern Med 2016;176:12861293.

2. Lavand'homme P, Thienpont E. Pain after total knee arthroplasty: a narrative review focusing on the stratification of patients at risk for persistent pain. Bone Joint J 2015;97-B (10 Suppl A):45-48.

3. Chodor P, Kruczynski J. Predicting persistent unclear pain following primary total knee arthroplasty. Ortop Traumatol Rehabil 2016;18:527-536.

4. Brummett CM, Waljee JF, Goesling J, Moser S, Lin P, Englesbe MJ, et al. New persistent opioid use after minor and major surgical procedures in US adults. JAMA Surg 2017;152:e170504.

5. Patanwala AE, Jarzyna DL, Miller MD, Erstad BL. Comparison of opioid requirements and analgesic response in opioid-tolerant versus opioid-naïve patients after total knee arthroplasty. Pharmacotherapy 2008;28:1453-1460.

6. Namba RS, Singh A, Paxton EW, Inacio MCS. Patient factors associated with prolonged opioid use after total knee arthroplasty. J Arthroplasty 2018; doi 10.1016/j.arth.2018.03.068.

7. Waljee JF, Zhong L, Hou H, Sears E, Brummett C, Chung KC. The Use of Opioid Analgesics following Common Upper Extremity Surgical Procedures: A National, Population-Based Study. Plast Reconstr Surg 2016;137:355e-364e.

8. Carroll I, Barelka P, Wang CK, et al. A pilot cohort study of the determinants of longitudinal opioid use after surgery. Anesth Analg 2012;115:694-702. 
9. Goesling J, Moser SE, Zaidi B, et al. Trends and predictors of opioid use after total knee and total hip arthroplasty. Pain 2016;157:1259-1265.

10. Kain Z, Vakharia S, Garson L, et al. The perioperative surgical home as a future perioperative practice model. Anesth Analg 2014;118(5):1126-40.

11. de Hoogd S, Ahlers SJ, van Dongen EP, et al. Is intraoperative remifentanil associated with acute or chronic postoperative pain after prolonged surgery? An update of the literature. Clin J Pain 2016;32:726-35.

12. Chia YY, Liu K, Wang JJ, Kuo MC, Shung-Tai H. Intraoperative high dose fentanyl induces postoperative fentanyl tolerance. Can J Anesth 1999;46:872-877.

13. Hayhurst CJ, Durieux ME. Anesthesiology 2016;124:483-8.

14. Mcfarlane AJR, Prasad GA, Chan VWS, Brull R. Does Regional Anesthesia Improve Outcome After Total Knee Arthroplasty? Clin Orthop Relat Res 2009;467:2379-2402.

15. Smith LM, Cozowicz C, Uda Y, Memtsoudis SG, Barrington MJ. Neuraxial and combined neuraxial/general anesthesia compared to general anesthesia for major truncal and lower limb surgery: a systematic review and meta-analysis. Anesth Analg 2017;125:1931-1945.

16. Helwani MA, Avidan MS, Abdallah AB, Kaiser DJ, Clohisy JC, Hall BL, et al. Effects of regional versus general anesthesia on outcomes after total hip arthroplasty. J Bone Joint Surg Am 2015;97:186-93.

17. Webb CA, Mariano ER. Best multimodal analgesic protocol for total knee arthroplasty. Pain Manag 2015;5:185-196.

18. Kehlet H, Mogensen T. Hospital stay of 2 days after open sigmoidectomy with a multimodal rehabilitation programme. Br J Surg 1999;86(2):227-230. 
19. Miller TE, Thacker JK, White WD, Mantyh C, Migaly J, et al. Reduced Length of Stay after Implementation of an Enhanced Recovery Colorectal Protocol. Anesth Analg 2014;118:10521060

20. Thiele RH, Rea KM, Turrentine FE, Friel CM, Hassinger TE, et al. Standardization of Care: Impact of an Enhanced Recovery Protocol on Length of Stay, Complications, and Direct Costs after Colorectal Surgery. JACS 2015;220(4):430-443.

21. Scott NB, McDonald D, Campbell J, Smith RD, Carey AK, et al. The use of enhanced recovery after surgery (ERAS) principles in Scottish orthopaedic units - an implementation and follow-up at 1 year, 2010-2011: a report from the Musculoskeletal Audit, Scotland. Arch Orthop Trauma Surg 2013;113:117-124.

22. Gustafsson UO, Hausel J, Thorell A, Ljungqvist O, Soop M, et al. Adherence to the Enhanced Recovery After Surgery Protocol and Outcomes After Colorectal Cancer Surgery. Arch Surg. 2011;146(5):571-577.

23. Ahmed J, Khan S, Lim M, Chandrasekaran TV, Macfie J. Enhanced recovery after surgery protocols - compliance and variations in practice during routine colorectal surgery. Colorectal Dis. 2012;14(9):1045-51.

24. Mariano ER, Vetter TR, Kain ZN. The Perioperative Surgical Home is Not Just a Name. Anesth Analg. 2017;125(5):1443-1445.

25. Edlund MJ, Martin BC, Russo JE, DeVries A, Braden JB, et al. The Role of Opioid Prescription in Incident Opioid Abuse and Dependence Among Individuals with Chronic Noncancer Pain: the Role of Opioid Prescription. Clin J Pain 2014;30:557-64. 
26. Shah A, Hayes CJ, Martin BC. Characteristics of initial prescription episodes and likelihood of long-term opioid use - United States, 2006-2015. MMWR Morb Mortal Wkly Rep 2017;66:265-269.

27. Centers for Disease Control and Prevention. Annual Surveillance Report of Drug-Related Risks and Outcomes-United States, https://www.cdc.gov/drugoverdose/pdf/pubs/2017-cdcdrug-surveillance-report.pdf; 2017 [accessed June 1, 2017].

28. Levy B, Pulozzi L, Mack KA, Jones C. Trends in Opioid Analgesi - Prescribing Rates by Specialty, U.S., 2007-2012. AJPM 2015;49(3):409-413.

29. Drug Enforcement Administration. Established Aggregate Production Quotas for Schedule I and II Controlled Substances and Assessment of Annual Needs for the List I Chemicals Ephedrine, Pseudoephedrine, and Phenylpropanolamine for 2018, https://www.deadiversion.usdoj.gov/fed_regs/quotas/2017/fr1108.htm; 2017 [accessed June 1, 2017]

30. CVS Health. CVS Health Fighting National Opioid Abuse Epidemic With Enterprise Initiatives, https://cvshealth.com/newsroom/press-releases/cvs-health-fighting-national-opioidabuse-epidemic-with-enterprise-initiatives; 2017 [accessed June 5, 2017].

31. Welchek CM, Mastrangelo L, Sinatra RS, Martinez R. Qualitative and Quantitative Assessment of Pain. In: Sinatra RS, deLeon-Casasola OA, Ginsberg B, Viscusi ER, eds. Acute Pain Management. Cambridge University Press, New York: 2009; pp. 147-166. 